\title{
A Blockchain-Based Approach Towards Overcoming Financial Fraud in Public Sector Services
}

\author{
Hissu Hyvärinen · Marten Risius · Gustav Friis
}

Received: 30 December 2016/Accepted: 11 September 2017/Published online: 3 November 2017

(C) Springer Fachmedien Wiesbaden GmbH 2017

\begin{abstract}
In financial markets it is common for companies and individuals to invest into foreign companies. To avoid the double taxation of investors on dividend payment both in the country where the profit is generated as well as the country of residence - most governments have entered into bilateral double taxation treaties, whereby investors can claim a tax refund in the country where the profit is generated. Due to easily forgeable documents and insufficient international exchange of information between tax authorities, investors illegitimately apply for these tax returns causing an estimated damage of 1.8 billion USD, for example, in Denmark alone. This paper assesses the potential of a blockchain database to provide a feasible solution for overcoming this problem against the backdrop of recent advances in the public sector and the unique set of blockchain capacities. Towards this end, we develop and evaluate a blockchain-based prototype system aimed at eliminating this type of tax fraud and increasing transparency regarding the flow of dividends. While the prototype is based on the specific context of the Danish tax
\end{abstract}

Accepted after three revisions by the editors of the special issue.

H. Hyvärinen $(\bowtie)$

Department of Business IT, IT University of Copenhagen,

Rued Langgaards Vej 7, 2300 Copenhagen, Denmark

e-mail: hissu@itu.dk

Dr. M. Risius

College of Business and Behavioral Science, Clemson

University, 515 Calhoun Dr, Room 137, Clemson,

SC 29634, USA

e-mail: risius@g.clemson.edu

G. Friis

Brainbot Technologies AG, Mainz, Germany

e-mail: grbf@icloud.com authority, we discuss how it can be generalized for tracking international and interorganizational transactions.

Keywords Blockchain - Public services sector - Taxation · Double spending · Double taxation treaties · Design science

\section{Introduction}

In the globally interconnected financial markets, a growing number of investors earn taxable gains (e.g., dividends, profits) in countries where they do not legally reside. In order to avoid taxing investors both in the country of residence as well as in the country where the profit is generated, many nations enter into bilateral double taxation treaties (DTTs) with each other, whereby the country in which the profit was made deducts a withholding tax at the source (e.g., the company paying the dividend) and the investor can claim a respective foreign tax credit in the country of residence (PWC 2016). However, illegitimate tax credit compensations are difficult for tax authorities to control because investing companies can be simultaneously located in multiple countries and individuals can easily forge residency documents (PWC 2015). Moreover, whereas the majority of countries worldwide have ratified DTTs, the treaties have, in general, not been coupled with an infrastructure enabling the exchange of personal investor information between taxation authorities. As a result of this lack of systematic information exchange, several countries have fallen victim to criminal actors submitting fraudulent tax refund applications. Institutional banks have allegedly even offered so-called cumcum trades as services to their clients, allowing foreign investors to profit from tax breaks of national shareholders on their dividends (Matussek 2016). One example is the Danish Tax Authorities (SKAT) which suffered an estimated 
loss of 1.8 billion USD through forged dividend tax refund applications by the end of August 2015 (Skatteministeriet 2016). The loophole in the Danish approval process can arguably be characterized as a "double spending" problem, enabled by the sole reliance on national and stand-alone registry systems. In essence, it is a system that provides neither the transactional nor geographical proof required to make an informed decision on the applicant's entitlement to a tax refund.

Currently there is no central information system dedicated to managing the flow of information between involved parties in order to reliably check an applicant's eligibility for a tax refund. Thus, in order to deal with the double spending issue, taxation authorities could implement a new database that would offer support for managing international tax claims. In light of the blockchain applicability analysis framework (Glaser 2017), we assume this double spending problem presents a viable use case for a blockchain database. The current absence of an alternative system to track the questionable cross-border dividend flow substantially increases the feasibility of a blockchain-based solution, because the common reservations regarding legacy systems (i.e., the trade-off between running outdated maintenance-intensive systems and the expensive implementation of a new system) would not apply (Bennett 1995) in this case. Furthermore, its technologically open qualities and general pervasiveness (extending from backend database systems through business logic entities, up to organizational layers) (Glaser 2017) make blockchain technology well-suited as a comprehensive solution to double spending vis-vis building and integrating a traditional database system. Due to its immutable log of historical transactions, a private permissioned blockchain system as a distributed ledger technology could offer a viable solution for auditing purposes (Glaser 2017). Furthermore, properly coded blockchain-based transactions on Ethereum, for example, have been shown to be potentially resistant to double spending problems (Natoli and Gramoli 2016). Finally, since smart contract execution reduces the required amount of external intervention (e.g., manually triggering token transfers along the dividend dissemination process), blockchain could minimize expenses.

Thus, in this study we strive to investigate the suitability of using blockchain technology - as opposed to a traditional database system - for overcoming the loophole in the Danish dividend-tax refund system described above. Therefore, we follow a design science approach aimed at developing and evaluating a prototype for a blockchain-based solution that allows the dividend flow to be traced. The prototype is designed to assist in verifying if an individual is entitled to a tax refund, and to overcome the current practical approval deficiencies by also facilitating the informational exchange between tax authorities. In general, this study investigates whether and how a blockchain-based system could improve the exchange of information in the public sector for the purpose of eliminating tax fraud.

As such, we provide practical evidence for the potential of blockchain technology in overcoming current taxation issues. Furthermore, this research also pertains to the more general context of public registry systems. As argued by the United Kingdom's Government Office of Science, ledger technology has the potential to transform the way a range of public services are delivered, such as collecting taxes, delivering benefits, issuing passports, recording land registries, assuring the supply chain of goods, and generally ensuring the integrity of government records and services (Government Office for Science 2016). This research aims at assessing the applicability of blockchain technology for the public service industry.

The remainder of the paper is structured as follows. In Sect. 2, we further discuss the double spending problem and briefly introduce the core blockchain features and related elements that are relevant to eliminating this type of tax fraud. Section 3 explains the design science process and decisions as well as the artifact that we built, which is critically evaluated in Sect. 4. Section 5 critically discusses the study's findings, while Sect. 6 offers the conclusions reached by our study as well as its limitations and opportunities for future research.

\section{Theoretical Background}

The goal of this paper is to develop a potential solution for overcoming governmental taxation issues related to the aforementioned double spending problem of the Danish tax authority. In light of the application requirements, we assume that the double spending issue presents a potential use case for a blockchain database (Glaser 2017). To test this assumption, however, we must first revise the current usage of blockchain technologies in the public sector and then link the specific case requirements to the technological blockchain applications in order to assess its legal and technical potential for resolving the double spending issue.

\subsection{Blockchain Use Cases in the Public Sector}

With the legacy of Bitcoin in mind, blockchain has traditionally been perceived as a rather marginal technology, used mainly by a small, tech-savvy group of people. This general perception is increasingly changing due to the opportunities offered by interoperable next-generation blockchains, reflected by heavy investment by financial institutions and venture capital funds. As such, blockchain applicability is often discussed in the financial sector regarding securities issuance, insurance, trading and 
settlement (Beck and Müller-Bloch 2017; Nofer et al. 2017). Quite recently, the potential impact of the blockchain technology applied to the public sector has begun to be recognized by public agencies, governments and industry providers. Consequently, the United Kingdom's Government Office of Science states that blockchain applied to the public sector has the potential to (1) enhance the protection of critical infrastructure and data, (2) reduce operational costs, and (3) facilitate transparency and the traceability of transactions (Government Office for Science 2016). Accordingly, the Estonian Government, for example, took action in early March 2016 to build a prototype to store one million individual health care records on a distributed ledger (Williams-Grut 2016). Similarly, Honduras and Georgia have also reportedly been experimenting with distributed ledger technologies in order to improve the national public land-registries (Epstein 2015). In the specific case of Denmark, public authorities have even considered introducing a blockchain-based equivalent to the national currency in order to save costs and facilitate small and micro electronic payments (Carlström 2016). Such implementations of blockchain technology are clearly beneficial for secure, cost-effective, and tamper-proof national registries. Furthermore, being transparent and traceable is important so that public agencies can approve and monitor public spending. Specific cases analyzed by the UK government include how to approve and distribute welfare support in order to avoid fraud and errors leading to misappropriation of funds. One of the cases regarding fraud and error in distributing benefits illustrates how assigning citizens with digital identities improves the exchange of information among public agencies for the purpose of managing eligibility; it also allows citizens without bank accounts to receive public financial support directly on any device capable of storing an electronic currency wallet (Department for Work and Pensions 2013). Further public service use cases related to financial issues address individual identification (e.g., to prevent money laundering), pretransaction processes (i.e., creating, validating, and transmitting payments), clearing and settling transactions as well as postsettlement (e.g., reconciliation, reporting transactions, contract enforcement) (Bank of International Settlements 2017).

The benefits of using blockchain technology to improve informational flow among public agencies and stakeholders have also been discussed in an international and multistakeholder context, in which it has been argued that disintermediation in terms of cutting the middleman out of the process is supported through blockchain technology and could function in various ways when providing public services. On the one hand, blockchain solutions enable bypassing local banks or even local governments in the case of international aid transactions made by individuals, public agencies, and NGOs. This means that aid can be sent directly to the receivers in a peer-to-peer format, without having to go through local banks or authorities (Government Office for Science 2016). This facilitates, for example, a reduction in fees since currency restrictions are bypassed, and it provides information to the sender as to how the donation has been spent. It can even give the sender the capacity to decide what services the donation can be used for. On the other hand, public agencies can also communicate directly from one institution to another, disintermediating the citizen, whose only role would then be to trigger an exchange of information by applying for a public service. Therefore, institutions would no longer have to rely on the validity of the information provided by the individual.

Such a system has been proposed by the United Kingdom's Government Office of Science as a suitable solution for establishing a shared European value added tax system (VAT). The proposal involves creating a unilateral system tracking all VAT eligible transactions, while accommodating differences in VAT applicability across the member states. With the financial institutions and citizens on board, the system would facilitate an international exchange of information capable of preventing the annual €151-193 billion loss resulting from VAT fraud (Eureporter.co 2013). While the UK government assesses the solution as technologically mature, the success of making blockchainpowered exchange of international information a reality in the public sector hinges on political alignment and industry participation (Government Office for Science 2016).

Although these fields of application have been identified by respective experts, no working prototypes have been introduced. Introducing new systems, especially prototypes based on a novel technology such as blockchain commonly raises legal concerns (Bank of International Settlements 2017). However, we feel confident that the aforementioned use cases demonstrate the potential of blockchain to accommodate current legal standards. To critically evaluate the potential technological applicability of blockchain in the public service sector, we next describe the relevant blockchain affordances in order to investigate its technical suitability for this case.

\subsection{Related Blockchain Properties}

We decided to use a blockchain-based approach, because blockchain offers several features that are particularly useful for overcoming the issue of double spending described above.

Blockchain is an emerging technology which was originally used to implement cryptocurrencies (Nakamoto 2008). While blockchain has become known as the technology behind Bitcoin, it is not limited to financial exchanges; rather, it can be used for transactions in general 
without involving an intermediary. Examples of potential application areas pertain to digital assets, marketplaces, notary services (Korpela et al. 2017; Wörner et al. 2016), supply chain information (Korpela et al. 2017), and energy (Aitzhan and Svetinovic 2016) and healthcare sectors (Mettler 2016). While it is often claimed that it is a technology with substantial disruptive economic potential, the design science approach from Beck et al. (2016) constitutes the first scientific approach modeling potential economic implications of these systems, and the case study by Beck and Müller-Bloch (2017) is the first academically published analysis on how incumbent organizations such as banks deal with innovation related to blockchain.

Reduced to its essentials, blockchain is a distributed, transactional database with distributed nodes linked by a peer-to-peer network. Each node in the network contributes to verifying the transactions and sends information about them to the other nodes via their public key. Nodes identify each other by the IP address, while users reference each other via their public keys (Tschorsch and Scheuermann 2015). In the context of this study, every acting unit (e.g., SKAT, dividend recipient, intermediary banking organization) is an individual user, and some users also act as nodes.

Many blockchain systems support transferable tokens, either as an inherent feature or implementable in higherlevel scripting or programming languages (Glaser 2017). In the original case, these tokens are treated as a coin to be transferred between nodes (Yli-Huumo et al. 2016). In the meantime tokens have expanded from being conceived of as a simple coin to becoming a representation of property, utility, rewards, or fungibility (LeBeau 2017). Tokens have distinct properties depending on their purposes. Therefore, different blockchain platforms host different tokens (Ethplorer 2017).

Smart contracts manage tokens that represent, for example, the account balance of a particular user address stored on the blockchain. When transferring tokens, smart contracts enter the appropriate number of tokens into a local database containing information on the amount and the user address (Kosba et al. 2016). This process is systematically equivalent to transferring funds into a bank account. Ultimately, these tokens can be maintained autonomously by the rules specified in the smart contract.

In operational terms, blockchain as a database comprises an event log where transactions or other events are stored such that they are immutable after having been submitted to the system. Rather than being stored in a database on a central server, a copy of the data exists on each node participating in the blockchain (Yli-Huumo et al. 2016). Each block in a blockchain contains a link to the previous block in the chain, a proof-of-work element, and a listing of one or more transactions. The link to the previous block is encrypted by using a hash function for the transaction part of the previous block (Tschorsch and Scheuermann 2015). This allows information from previous blocks to be stored in successor blocks in nonlegible form. Linking the blocks together using hash references is a way of preventing "bad" blocks from being inserted unnoticed between legitimate ones, as that would break the easy-to-verify chain of hashes matching the content of the predecessor blocks. When transactions are broadcast to the network of nodes, each node competes to try to complete the block containing the transactions. Once the node has solved the hash - i.e., found the proof-of-work - it broadcasts the finished block to the other nodes, after which point that block cannot be changed without recomputing the proofof-work for that block and for every successor.

Finally, in the original conceptualization of blockchain (e.g., in Bitcoin), any transaction is visible to all participants, thus providing maximum transparency and replicability of transactions (Tschorsch and Scheuermann 2015).

\subsection{Blockchain Features Addressing the Double Spending Issue}

The aforementioned blockchain properties assist in overcoming the issue of double spending tax refunds in several ways. In this way, the commonly issued concern that blockchain could be a potentially disruptive technology in search of use cases is also addressed (Avital et al. 2016). The blockchain's core capacity to manage transactions is essential for our approach to preventing double spending. In the context of electronic payment systems, double spending occurs when several transactions are created for the same unit(s) of currency. Because the chronological order of transactions is verified through computational proof on the blockchain, double spending is extremely unlikely (Nakamoto 2008). Since the earlier days of Bitcoin, several improvements have been implemented in blockchain systems to further ensure the failure of double spending attacks (Karame et al. 2012; Rosenfeld 2014; Sompolinsky and Zohar 2015). In the case presented in this paper, the double spending problem arises from a lack of monitoring and information rather than a technical failure; more than one person can apply for the same tax refund on a dividend without being detected. Implementing the process on a blockchain would ensure that no double spending situations can occur, and that the payments are traceable (Natoli and Gramoli 2016).

In order to conduct and track dividend payments on the blockchain, tokens can be used to represent the dividend originally issued by a company. Thus, the system tokens would receive official value backing comparable to traditional binding forms - for example, database entries representing commercial bank money. Guaranteeing the 
economic value of these system tokens is essential for overcoming the critical mass issue regarding the likelihood with which users would adopt a blockchain system, as it holds out the prospect of real economic value (i.e., a tax refund).

Managing this token dissemination process definitively necessitates trust in the token issuing (i.e., VP Securities, a financial services company reporting dividend payments to SKAT) and refunding institutions (i.e., SKAT), as well as the external input from token transferring entities (e.g., banks). The design solution that this paper seeks is a smart contract that maps dividend payments and promises low maintenance costs due to automated execution of smart contract algorithms (Bank of International Settlements 2017). Blockchain is often credited with the ability to decentralize control through its consensus mechanism between the participating nodes in the system (Nakamoto 2008; Pilkington 2016). While this holds true for the autonomously operating smart contract itself, the decentralization of control ends at the boundaries to the SKAT system, which exchanges the system token into a valuable currency. While one could imagine a scenario in which an entire economic ecosystem being integrated into this blockchain system, the chances of such an evolution occuring seem unrealistic. Thus, while a smart contract itself is decentralized and autonomous, the integration into existing payment processes guarantees value only if trust exists among the involved institutions.

The cryptographically linked transaction log makes the blockchain resistant to manipulation (Gervais et al. 2016). This immutability of logs proposes a blockchain system as a paramount solution for auditing purposes, as is necessary in the case of double spending. The transparency commonly associated with blockchain is an unacceptable property in an context involving highly sensitive data and tax refunds. In blockchain-related environments particular measures often must be undertaken in order to guarantee data privacy of users (Fabian et al. 2016).

Thus, this environment would require a permissioned blockchain, where only a specific set of permissioned users (i.e., SKAT) can see and validate transactions. In this case, privacy issues would not be a problem since SKAT - as the permissioned party - would see the content in the traditional database setup as well.

In sum, we assume that a blockchain-based solution is technically as well as legally feasible and offers some key advantages compared to a traditional central database system for solving the double spending issue. Technical feasibility becomes apparent in light of the blockchain applicability analysis framework (Glaser 2017). Accordingly, a blockchain-based solution is applicable since the tax environment represents a collaborative market requiring commercial value to be linked through trusted interfaces to provide a public good. Considering the aforementioned advances of blockchain in the public sector, it seems that legal constraints regarding, for example, data privacy can be accommodated by blockchain systems. Furthermore, the current absence of a system to manage international tax refunds presents a need for the implementation of a new system. The pervasive structure of blockchain databases offers a comprehensive solution that is easily accessible for end users and can be rapidly integrated into existing banking and tax authority systems. Moreover, smart contract execution requires very limited external and manual involvement, which suggests that a blockchain system may be more efficient compared to traditional database systems. Finally, the immutable log of past transactions constitutes an irrefutable advantage of blockchain databases over traditional counterparts for auditing purposes. It is important that tax authorities have the ability to track tax refund entitlements in order to prevent banks and individuals from paying or receiving fraudulent or otherwise erroneous claims. In a traditional database, banks can report having paid any amount at any point along the multistep dividend dissemination process (see Fig. 1) with no simple way of formally tracing whether a dividend has actually been paid. In the case of discrepancies between claimed and paid dividends, it would

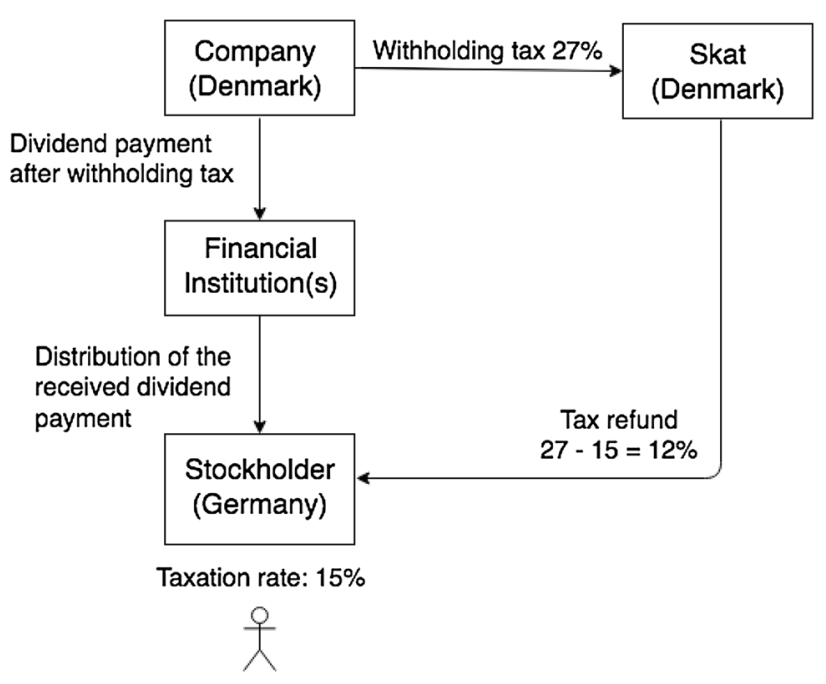

Fig. 1 An example of the tax refund process for dividend payments for stockholders living abroad. When a company pays dividends, they withhold the dividend tax paid to SKAT and pay the net dividend to the stockholders or the intermediary financial institutions representing the stockholders. The financial institutions then pay the dividend onwards so that it reaches the stockholders. The chain of payment may contain several financial institutions as intermediaries that are not necessarily in the same country as the stockholder or the company. If a stockholder lives in a country with a lower tax rate than Denmark, they are eligible for a tax refund worth the difference between the taxation rates between Denmark and the country in which they pay taxes. The percentages used in this figure are examples for the sake of illustration 
require quite a lot of effort (and is arguably even impossible) to retrace the global interorganizational flow of payments in the case of fraudulent or erroneous reports in order to identify the source of the error. Blockchain, however, enables the transparency and traceability of transactions throughout the dividend dissemination chain from the point of payment to the final recipient.

\section{The Design of the Artifact}

In this section, we briefly describe the problem faced by Danish tax authorities regarding tax refunds on dividends, explain how our design process addresses this issue, and document the prototype built to evaluate the design.

\subsection{The Danish Double Spending Problem}

Dividend tax is an income tax paid on dividend income received by the stockholders of a company. When a publicly traded company in Denmark pays out dividends, it withholds the tax - typically $27 \%$ - from the dividend and pays it to SKAT, the Danish tax authority. The companies are also obliged to report the dividend payments to VP Securities - a financial services company that provides securities and investor services for investors and organizations, which is also responsible for reporting the paid dividends to SKAT. If a stockholder lives - or more precisely, pays taxes - in a country that has a lower income tax than Denmark's, they are eligible to apply for a tax refund comprising the difference between Denmark's tax rate and that of the country in question. For instance, if a stockholder of a Danish company lives in Germany where the income tax is $15 \%$, they are entitled to a refund worth $12 \%$ of their gross dividend (example illustrated in Fig. 1.)

To prove their eligibility for a tax refund, the applicant must provide SKAT with a bank statement proving the dividend has been paid to them as well as a statement from their tax authority confirming they pay taxes to the country in question. In the current system, the documentation is delivered in paper form. This causes a significant amount of manual labor for the SKAT employees processing the request, which is why SKAT is interested in digital solutions to reduce the effort required by the process. In addition to being resource intensive, the current system opens the possibility of fraudulent applications, since it is the applicant who is responsible for providing the documentation proving their eligibility, and the process does not monitor of the flow of the dividend payment. There is no control mechanism in place to prevent several refund applications being submitted for the same dividend payment, which makes it possible for applicants to apply for already refunded dividend payments and use forged bank statements to justify their application. This was identified as the biggest issue with the current procedure from SKAT's point of view by the taxation experts interviewed for the evaluation of our design (see Sect. 4.3). Not being able to control for the eligibility of the applicants makes it more likely that a double spending situation where SKAT pays multiple refunds for the same dividend taxes will arise. Fixing the flaw in the current process would prevent significant future losses for SKAT.

\subsection{The Design Process}

In terms of design science, the double spending that occurs when refunding dividend taxes is a typical "wicked problem" since (1) it may only be possible to find a solution to the problem that is "good enough", rather than solving it completely; (2) the solution to the problem will be good-orbad rather than true-or-false; (3) testing the solution is complicated and depends on several contributing actors; (4) the possibility to learn by trial-and-error is limited as every attempt at testing the solution is complicated and resource intensive; and (5) the problem does not have an exhaustively describable set of potential solutions or a set of well-described permissible operations. We therefore chose the ad hoc development approach by first learning about the problem and then designing a draft, which we concurrently and conclusively evaluated. Therefore, our design process follows the DSRM Process Model introduced by Peffers et al. (2007), see Fig. 2 .

In our case, the research entry point was client initiated; SKAT approached us with the aforementioned double spending problem regarding dividend payments. The general problem is that SKAT loses a large amount of money annually due to fraudulent tax refund applications and double spending situations, which could be avoided by designing an artifact to facilitate reliable tracking of dividend payments abroad and international cross-institutional informational exchange. Based on the understanding of the problem acquired through conversations with SKAT representatives, we designed the Dividend Payment Control System described in Sect. 3.4. In addition to the initial conversation before commencing the design process, we also had several other meetings with SKAT, in which we presented our design and received feedback that lead to various alterations and improvements. After completing a prototype, we ran a simulation of a simple use case to demonstrate how our system would work. We evaluated our system using the FEDS framework (Venable et al. 2016) (see Sect. 4), guided by input from the taxation experts to make sure the developed prototype properly addresses the apparent problem from the perspectives of both SKAT and their clients. Finally, this project was publicly communicated to an audience of relevant experts 


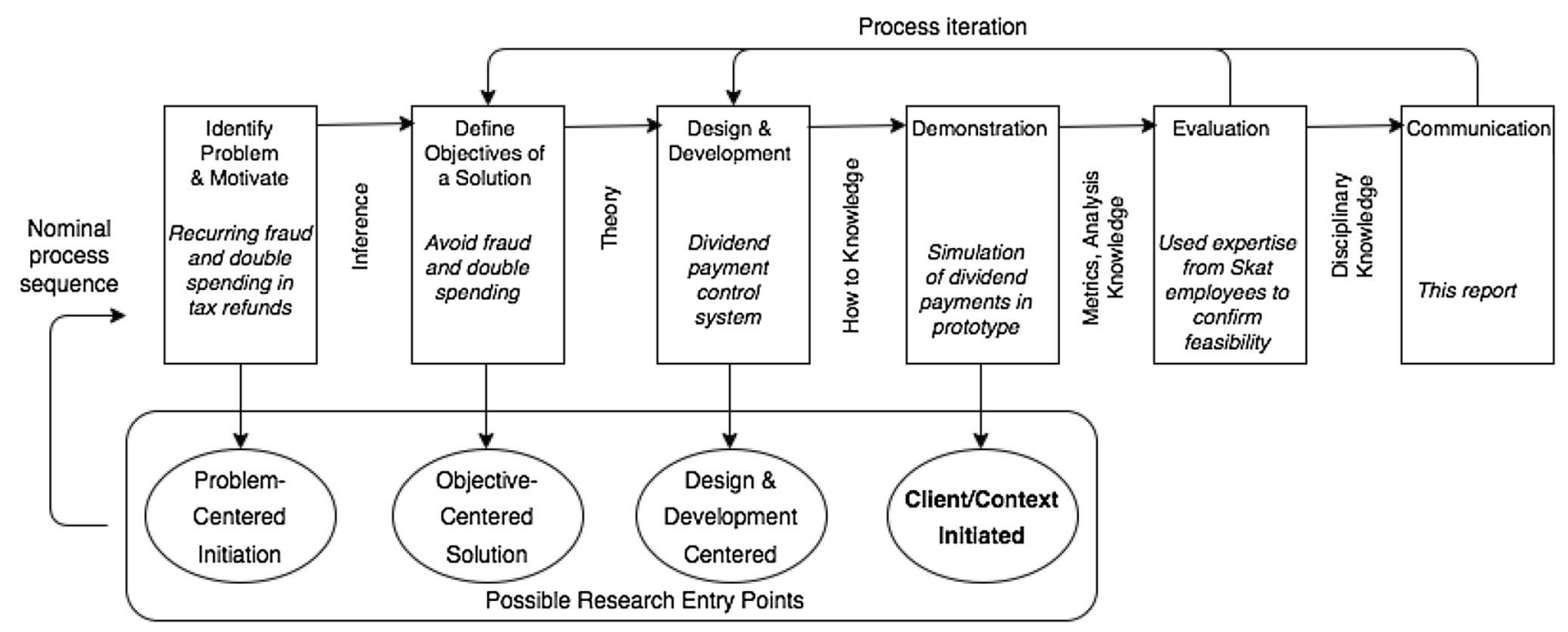

Fig. 2 The design science research process

from both science and industry at the Blockchain Summer School 2016 held in Copenhagen, Denmark (http://block chainschool.eu), which provided the basis for this report.

On a higher level of abstraction, this process is characterized by the three-cycle view introduced by Hevner et al. (2004): SKAT represents the environment where business needs arise, while existing knowledge about blockchain technology and its application areas represents the applicable knowledge base. We made iterations through the first cycle of developing and evaluating by utilizing the feedback from SKAT during our design process.

\subsection{Design Decisions}

The first step in our design process was to establish the requirements for an improved dividend tax refund system. The most important demand was to solve the double spending problem, which was one of the main reasons for SKAT's dissatisfaction with the current process. The other important requirements were related to ease of use by different actors involved with the process. The system should not introduce major changes in the roles of VP Securities, the financial institutions, or stockholders. Additionally, the system should be convenient from the perspective of the stockholders in order to avoid discouraging them from making investments Danish company stocks. Finally, the system should reduce the amount of labor required by the SKAT employees, since the current process involves manually processing each piece of information that SKAT receives from each different actor.

Blockchain was chosen as the underlying technology as it supports multiple information contributors, guarantees immutability of transaction records, and ensures the prevention of double spending (i.e., several people fraudulently applying for a dividend tax refund). The smart contracts deployed on the Ethereum blockchain enabled us to implement a strongly automated token distributing system correspondent with the structure of the dividend payments. Thereby, the system facilitates tracing the flow of dividends and the exchange of supplementary documents in order to prove the consequent entitlement to a tax refund.

Due to the exploratory nature of this project, we decided to focus on implementing a functional dividend payment representation on the blockchain that could subsequently be expanded to more elements and actors. For an ultimately comprehensive system, the foreign tax authorities would also be included on the blockchain as actors like SKAT enabling them to confirm the residency of an tax refund applicant and to access information regarding the applicant's dividend income for tax purposes. Thereby, the blockchain-based solution would facilitate the data exchange between authorities in order to improve the informational deficiencies occurring in the current system.

\subsubsection{User Groups}

The users of the system comprise four categories: SKAT, VP Securities, the financial institutions, and the stockholders. Each category has a different role and thus different rights. As the organization distributing the refunds, SKAT would own the system and have full access to the data in it. VP Securities would have a special role as well; they would report the first step of the dividend payments and provide the information regarding amounts that have been paid out. This can be implemented using a smart contract that issues tokens if a payment is reported by VP Securities?the only user with the authority to create 


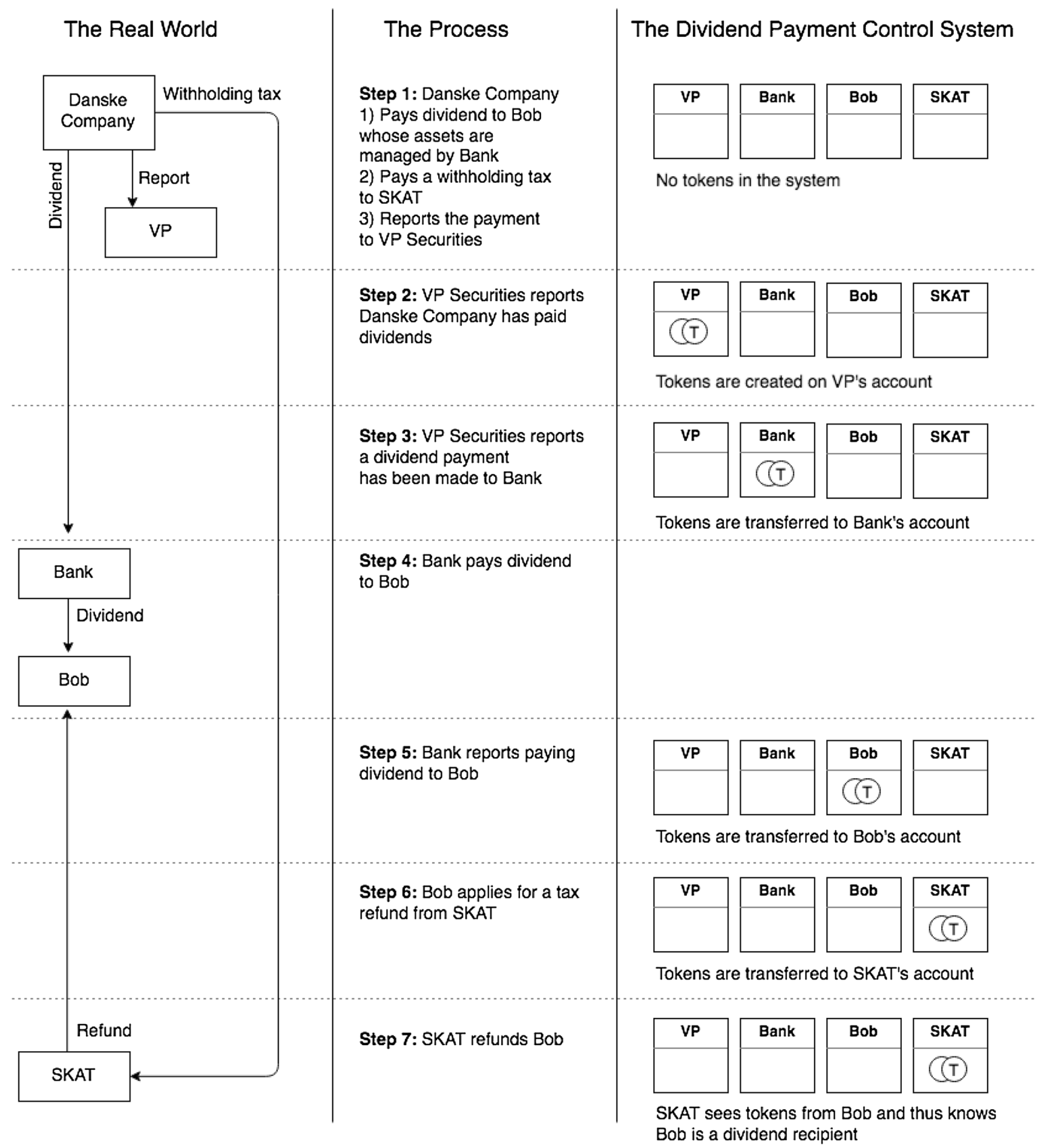

Fig. 3 The process from when a dividend is paid to when the stockholder receives a refund. The payments as well as the companies reporting to VP Securities (steps 1, 4 and 7) occur outside of the system. Reporting the payments and applying for the tax refund (steps

tokens?ensuring that the number of tokens generated in the system matches the paid dividend reported to them by the companies. VP Securities would not be able to access
2, 3, 5 and 6) are actions performed within the system. The right hand side demonstrates how the tokens are propagated from one account to another based on payment reports

transactions unrelated to their user account. Each financial institution and stockholder would have a user account that they open and manage themselves. The financial 


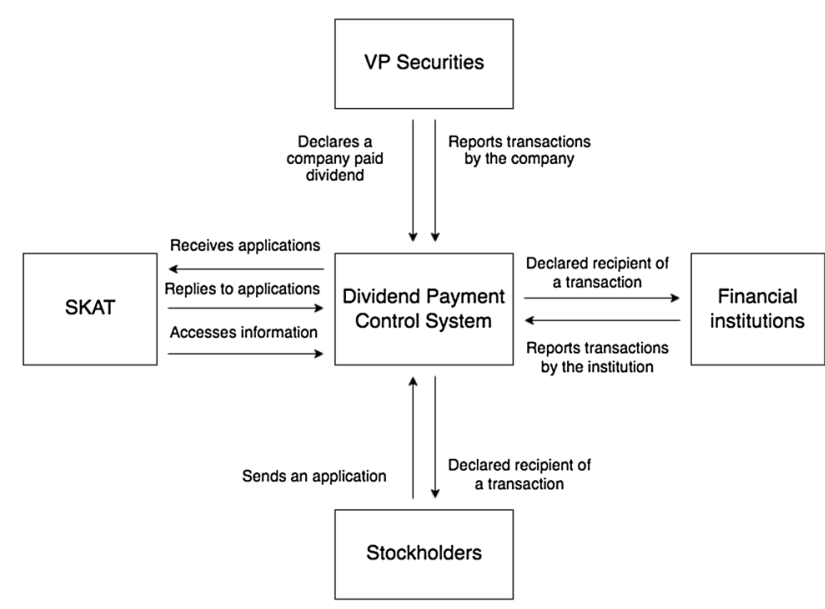

Fig. 4 An overview of the functionalities available to each user group in the system. The arrows towards the Dividend Payment Control System represent input provided by a user, and the arrows outwards represent output provided by the system to the user

institutions would use their accounts to report the dividend payments, and the stockholders could then apply for a tax refund from SKAT, with their eligibility confirmed by the traceable payments documented within the system. The correct amount of the tax return would be automatically calculated. Neither the stockholders nor the financial institutions would have access to information other than that pertaining to their own accounts. To summarize, while the system has a certain aspect of centrality, since it serves as a solution for different actors, who each provide different kinds of information to some central authority, the data entry and the mechanism of propagating the dividend payments are decentralized.

The SKAT feedback rounds informed us that financial institutions are obliged to collaborate with tax authorities regarding the disclosure of dividend payment processes. According to the SKAT representatives, any future improvement can assume collaboration from the financial institutions managing joint stockholder accounts. In order to make the dividend payment process traceable, the financial institutions are obliged to announce the payments if required by the tax authorities. Therefore our design is based on the assumption that the financial institutions comply with the request for participation. Involving them directly also has the advantage of removing the possibility of applicants forging bank statements for fraudulent refund applications.

Finally, we decided that each tax refund applicant should also obtain a user account on the blockchain to apply for refunds. Creating and managing an account on the blockchain is as convenient as filling out an application on a website, which fulfills the ease of use requirement. At the same time access to the blockchain enables the applicants to follow the progress of the dividend payment reports, improving the transparency of the process for them. Furthermore, this solution saves SKAT the effort of assembling and maintaining a applicant records, thus acknowledging the requirement of reducing SKAT's workload.

\subsubsection{User Authentication}

One issue with the current process relayed by SKAT during the interviews is that reliably identifying stockholders applying for refunds can present some difficulty. There are countries where citizens are not assigned a unique identifier such as a social security number. One significant advantage of the designed system is that the user IDs (or, more precisely, the public keys) associated with the stockholder accounts can be used as unique identifiers, confirmed by either VP Securities based on the information disclosed by the company paying the dividend (in cases where the dividend is paid directly to the stockholder) or a financial institution (if the payment process goes through intermediaries). The financial institutions would get the user account information along with the other personal details the stockholder relays to them as their client. This does not ultimately remove the possibility of a fraudulent bank confirming a fraudulent person, but providing some sort of unique ID is a significant improvement over the current situation and provides a means of processing users from various countries in a uniform fashion, thus simplifying the process. Additionally, being required to submit client identification on an immutable blockchain would likely act as a fraud deterrent, as evidence would be produced and the fraudulent actor could then be held accountable. Depending on SKAT's needs and preferences, the confirmation of a user could be either explicitly reported through a separate functionality of the system, or it could be implicitly included through the transferal of tokens to a user's account, which would thus serve as verification of the user for the payer.

\subsection{The Artifact}

The artifact in this work is the design of the Dividend Payment Control System (DPCS), which corresponds to the System Design category in the taxonomy of artifact types outlined by Offerman et al. (2010). The artifact was evaluated by building a prototype of the main technical functionality and by conducting expert interviews to assess the feasibility of the overall design.

The DPCS stores information about dividend payments on a permissioned blockchain. The system uses tokens controlled by smart contracts to represent dividends 
Table 1 The evaluation episodes

\begin{tabular}{|c|c|c|}
\hline Episode & Evaluation method & Property of interest \\
\hline \multirow[t]{2}{*}{ Episode 1} & Logical argument & Actual effectiveness \\
\hline & Expert evaluation & Perceived usefulness \\
\hline \multirow[t]{2}{*}{ Episode 2} & Expert evaluation & Actual effectiveness \\
\hline & & Perceived usefulness \\
\hline \multirow[t]{2}{*}{ Episode 3} & Prototype & Actual efficiency \\
\hline & Illustrative scenario & Actual effectiveness \\
\hline \multirow[t]{2}{*}{ Episode 4} & Expert evaluation & Perceived usefulness \\
\hline & & Perceived ease of use \\
\hline
\end{tabular}

distributed by companies to stockholders and intermediaries. The function of the tokens is to ensure that the amount of dividend paid and the amount of tax returns issued correspond to each other, preventing a double spending situation. The users of the system - with the exception of SKAT - do not need to understand or be aware of the tokens, they are simply a system-internal means of bookkeeping. Figure 3 illustrates the dividend refund application process, showing the actions corresponding to each step in both the real world and the system.

The different user groups (SKAT, VP Securities, the financial institutions, and the stockholders) have different rights, and each user has a user ID and an account. SKAT is the only user with full access to the information in the system as well as the ability to receive and respond to refund applications. The other user groups have one shared core functionality: declaring a transaction. VP Securities reports transactions made by a company to its stockholders, the financial institutions report transactions made by themselves to a stockholder (or, if there are several levels in the process, the next intermediary financial institution), and the stockholders apply for a tax refund, triggering a transaction of a corresponding number of tokens to SKAT. Whenever a transaction is reported, tokens are moved from the payer's account to the recipient's. In addition to declaring transactions, VP Securities has a special functionality for declaring a dividend payment event stating which company paid which dividend and how much was paid in total. The interaction of each user group with the system is illustrated in Fig. 4.

When a stockholder wants to apply for a tax refund, they create an account and send their account ID either to the financial institution managing their assets or - if they received the dividend directly from the company paying it out - to VP Securities. Once the transactions are reported and visible on the stockholder's account (meaning, from the system's point of view, that the tokens are there), they can apply for a tax refund which transfers the tokens to SKAT as proof of eligibility (and in order to ensure the tokens are removed from circulation once the tax refund has been paid out).

If a financial institution is an intermediary in a dividend payment process and their client wishes to apply for a tax refund from SKAT, the financial institution creates an account on the system (unless they already have one), relays their account ID to VP Securities, waits until the transaction from the company to the financial institution is reported to the system, and disburses the dividend payment (triggering the flow of a corresponding number of tokens), designating the client's account ID as the recipient.

When VP Securities reports a dividend payment event, they specify which company paid dividends and how much. This triggers a smart contract that automatically issues a number of tokens matching the dividend and places them into the account of VP Securities. If a stockholder receiving dividend payments directly from a company wishes to apply for a refund, they send their ID (public key) to VP Securities to enable the reporting of the transaction. Up to that point, the tokens stay on VP Securities' account.

SKAT's main use of the system is to receive tax refund applications after the user authentication and audit trail have already been resolved. SKAT can also access the transaction data if they wish to examine the chain of payment.

This blockchain-based approach provides several upsides for overcoming the current double spending issue. First, it significantly reduces the possibility for fraud since it documents the trace of the payment, meaning that applicants can no longer forge bank documents that would justify dividend payments as the banks themselves report the payments on the blockchain. Second, the approach eliminates the possibility of double spending since each token can only be used for a refund application once. Third, the blockchain solution makes it easy for SKAT to verify an applicant's right to a refund based on the tokens.

\section{Evaluation}

We used the Framework for Evaluation in Design Science Research (FEDS) (Venable et al. 2016) to guide the evaluation of the artifact. The design process was iterative, and during each design cycle improvements were made based on the evaluation results from SKAT experts in the previous cycle.

Our primary goal concerning the evaluation was to assess whether the artifact provides a feasible alternative to the current system by solving the double spending problem. The evaluation therefore focuses on uncertainty and risk reduction from both a technical (i.e., is the solution feasible and reliable?) and social (i.e., will the system be convenient enough for the users?) standpoint. 
The artifact was designed to be a component in a bigger, but yet undeveloped tax control system. Thus, practical evaluation with real users in the real world would not have been possible, which is why we chose an evaluation strategy with an emphasis on formative and artificial evaluation methods in terms of the "Technical Risk Efficacy" strategy (Venable et al. 2016). The properties our evaluation focuses on are actual effectiveness, actual efficiency, perceived usefulness and - to some extent - perceived ease of use (Moody 2003).

The design and evaluation process was divided into four episodes (see Table 1), each of which concentrated on one or two properties of interest that were evaluated using the most suitable method available, informed by the method types outlined by Peffers et al. (2012).

\subsection{Iterative Evaluation of the Effectiveness and Usefulness of the Design}

The first episode commenced with familiarizing ourselves with the problem and solution requirements; based on this episode, we designed a first draft of a model on paper. The main issues the tax authorities identified in Denmark's current dividend-refund payment system were fraudulent applications and the lack of information exchange regarding the dividend trail - both of these issues contributed to double spending situations - either due to deliberate fraud or to the applicants' insufficient understanding of tax laws in different countries. Thus, it became our main priority to design a solution that would prevent double spending and make fraud more difficult. In our structural analysis of the model we designed, we concluded that the model would resolve the double spending problem because of the blockchain structure and the properties of the smart contract we designed. During meetings with experts on the Danish tax system, we identified some inaccuracies and limitations in our model, which we proceeded to remedy during the subsequent phase.

During the second episode we improved the paper model based on the expert feedback received during the first phase. We then went back to the taxation experts to get their assessment of the usefulness of our design in solving the dividend refund problem. In addition, three blockchain experts were consulted regarding the feasibility and technical quality of the design, which resulted in some changes in the implementation.

\subsection{Experimental Use Case}

In episode 3, we built a prototype and designed an illustrative scenario in order to demonstrate the flow of the dividend payments and confirm the technical feasibility. The prototype was implemented by writing a smart contract and deploying it in the Ethereum Blockchain using the
Ethereum Wallet application (https://github.com/ethereum/ mist) as the graphical user interface. The smart contract controls tokens as a type of virtual currency that represents the dividend payments. Whenever a dividend payment between parties (e.g., company and investor, company and bank, bank and investor) is reported on the blockchain, a number of tokens corresponding to the amount transferred between bank accounts is moved from the payer's blockchain account to the receiver's. (See right hand side of Fig. 3.)

In our example scenario, a company (Danske Company) paid out dividends to a stockholder (Bob) through an intermediary financial institution (Bank 1). Accounts were created in the system for VP Securities, Bank 1 and Bob. The first step of the process was that VP Securities reported the dividend, causing Danske Company tokens to be created and placed on their account. They then verified that a transaction happened between Danske Company and Bank 1, which resulted in some of the tokens being transferred onto Bank 1 's account. Bank 1 then reported the dividend they paid out to Bob, triggering the transfer of the corresponding number of Danske Company tokens into Bob's account.

System users should not be able to report a dividend payment unless they have the corresponding tokens in their account. This is in order to prevent banks from reporting the same dividend payments multiple times or to different recipients; a form of fraud allowed by and present in the current application process. To demonstrate this, we created an account for an additional user, Bank 2. After receiving tokens from VP Securities, Bank 1 reported the dividend paid out to Bob, moving the tokens to Bob's account. Bank 1 then tried to verify the same sum being paid out to Bank 2 but failed, since they did not possess any more tokens.

After the tokens reach Bob's account, Bob can apply for a tax refund, which SKAT can now verify as legitimate by receiving the Danske Company tokens from Bob when he sends his application. Because the number of tokens matches the amount of dividend paid to the applicant, the correct amount of tax refund to be paid can be automatically calculated. Implementing the refund application process between Bob and SKAT was outside of the scope of the prototype, as the purpose was to demonstrate the usage of tokens to create the audit trail.

In addition to being communicated to an expert audience at the Blockchain Summer School, the design and prototype were presented at SKAT's request to a Danish cross-ministry working group charged with the task of charting out solutions for the dividend tax refund problem.

\subsection{Perceived Efficacy Based on Expert Feedback}

The fourth and final episode concentrated on evaluating the artifact's perceived efficacy, which is a combination of its 
perceived usefulness and perceived ease of use (Moody 2003).

For the purposes of evaluating our design, we consulted experts who were familiar with the dividend refund problem and had a good understanding of the technology used in our solution. We conducted semistructured interviews with two SKAT employees matching the aforementioned criteria. Both were familiar with the design and the prototype based on the communications of our work during the previous episode of the evaluation. The duration of the interview was a bit over half an hour in both cases. The interviews were conducted in English, recorded and transcribed. The questions asked were mostly open-ended, and were related to the design and usage of blockchain in general in the public sector from SKAT's perspective. The interview transcripts were analyzed using evaluation coding by two independent coders, and the codings were crosschecked to confirm consistency (Saldaña 2009).

When asked what the biggest issues with the current procedure are, both experts agreed it was the lack of traceability of the dividend payments, which makes it difficult to verify the eligibility of the refund applicant.

"The traceability of the dividends is the biggest issue." - Expert A

"We're not able to check if people are entitled to the refund they ask for." - Expert B

One of the experts also mentioned there is an overall lack of control in the system, creating potential losses for SKAT.

"There's no one-to-one system where what we get in taxes is the one that we pay out in tax dividend, so that kind of bottom line assurance that the two numbers are equal, we don't have that." - Expert B

Although there is a clear need for improving the situation, it was considered a priority to avoid making the refund process too inconvenient for the stockholders.

"Everyone is entitled for a refund if they received a dividend, even if we don't know about the path of the payment. The middle hands make it hard to track. However it's important to offer the refund possibility in order to prevent double taxation." - Expert A

"We also have this thing that we want people to invest in stocks in Denmark, so if you make a really efficient system where you have to prove a lot in order to get the refund, people will not invest if it becomes too difficult." - Expert B

The taxation experts concluded that the design solves the double spending and fraud problems in a satisfactory manner, which leads us to conclude the perceived usefulness of our design is appropriate and fulfils the requirement of solving the double spending problem. We also received confirmation that the problem addressed by the design was correctly understood.

"I think the solution pretty much solves the double spending. It also solves the fraudulence problem of end users making up claims that were never there. Your understanding of the problem was close to astonishing." - Expert A

When asked about whether it is feasible to assume compliance from the different actors involved in the system, the experts' view was that each group of actors has sufficient incentive to use the proposed solution because there were resource advantages (i.e., monetary, timewise, usability) or legal obligations for them.

"If we start with VP securities, we can pretty much force them to do whatever we want. It's a Danish company and they're providing an important service for the Danish financial sector so we are entitled to lay down rules that they have to apply." -Expert A

Regarding the financial institutions, several benefits were seen. They would likely be motivated to comply, since that would make applying for tax reductions easier for their clients, making them more lucrative service providers. In addition, it could also help them with their own goals regarding better traceability:

"That would be a service for them to give to the customers, to say hey I am on the blockchain which means it will be less of a hassle." - Expert A

"I think also the banks are interested in having a better overview, audit trail, because with all the scandals they're having now, with the Panama papers and stuff like that, they're actually spending a lot of money being able to know their customers." - Expert B

It was also pointed out that the design would save a great deal of effort from the person applying for the tax refund compared to the current arrangement:

"I can go to a bank that's on the chain and knowing that this one happened with a lot less effort, I would be automatically verified by my own tax administration and all the things that I normally would do in a manual way would be done for me. So I think there's incentive for the end user to make his purchase of shares where it is most easy to get a refund. If his investment has a size where a refund is important. The current system contains a lot of manual work for the end users, it's a major hassle." - Expert A 
The overall attitude towards adapting blockchain based solutions in the public sector was cautious but curious. When asked about factors discouraging the implementation of a system based on blockchain, the experts mentioned lack of knowledge, integrating data from legacy systems and the obscurity of legal aspects of the technology as concerns.

"The lack of knowledge of the topic at Skat. We'd need to hire someone who understands the technology and what the problems with applying it would be. Knowing where to start. There are many unknowns." - Expert A

"All the legal aspects of it, I mean that's a scary thing right, so we need to find a really small area where we could do some prototyping, and then build it up from there." - Expert B

SKAT is currently working on resolving the dividend refund issue by on the one hand making immediate, incremental changes to the current process, and on the other hand trying to find a better long-term solution; in addition to the cross-ministerial working group working on the problem, SKAT recently held a workshop to discuss ideas using our design as the baseline.

"[INTERVIEWER: What was the workshop about, what kinds of solutions did you discuss?] We started with your thoughts, so like the ground base for that, and then we tried to look deeper into, see how many, is it solvable, is it doable, and then I think one of the issues was the audit trail, like, you still have to add data to the system. And then we came up with some ideas, maybe we could just make $80 \%$ of it work in a blockchain, and we would know these are good and these are not good, so then we would definitely know what to look for." - Expert B

\section{Discussion}

This study evaluates the feasibility of a blockchain-based solution to overcome tax fraud as compared to traditional database systems. As such, we provide a blockchain-based design that enables tracking dividend payments from the issuer to the final recipient in order to overcome the issue of double spending and the lack of information available to the tax authorities. Although the developed system is not designed to be rolled out across tax authorities in different countries, it demonstrates a feasible solution to double spending, reduces the possibility of fraudulent tax refund applications, and automatizes a great part of the work previously conducted manually by SKAT employees.
While the investigated use case is limited to tax fraud, we assume that an adapted solution of our prototype could also be useful for avoiding fraud in existing intermediary banking systems (i.e., bank transaction and account managing systems) that companies utilize for various services.

Considering the close collaboration with and positive feedback from SKAT on our prototype, we feel confident that the developed system represents a viable solution to the complex issues of double spending. However, while our system contains major benefits of a blockchain-based solution (i.e., decentralization, transparency, immutability, automation) it cannot be considered an entirely trust-free system as it requires the compliance of banks and trust of the institutions that issue the tokens (i.e., VP securities) and refund taxes (i.e., tax authorities). This is, however, not a newly introduced feature of our solution but a requirement imposed by the established processes. As Glaser (2017) mentions, this type of trust is common when linking blockchain tokens to traditional monetary ecosystems. At the same time, however, this connection helps overcome the closed system of the respective blockchain environment by linking the digital tokens to commercial value. It should, however, be noted that within the proposed solution the vulnerability still exists that a financial institution could apply for a tax refund if the stockholder whose account they are managing fails do so. However, this issue already exists in the current system of dividend tax refunds, and even if this were to happen, the worst case scenario within our blockchain solution would be that SKAT would still distribute the refund amount that was originally issued as a dividend payment, because refunds would only be paid in exchange for tokens. Thus, the proposed system limits the currently rampant fraud to, at most, the amount of the issued dividend, which SKAT experts consider to be a major improvement over the current situation. Moreover, if this were to happen, the financial institution would risk being caught for fraud if the stockholder applied for the refund at some later point in time. In that case, it would be revealed that the tokens never arrived from the financial institution to their account and it could be verified which particular employee was responsible, because this information is all documented and traceable on the blockchain.

An additional point of discussion would be whether end users and financial institutions would be willing to adopt and comply with the system. For blockchain environments - as for any multisided market - a critical mass of customers and service providers must be attained in order to establish the closed nature of the system (Glaser 2017). According to SKAT it is reasonable to assume the cooperation of financial institutions, since they are obliged to comply when demanded to by governmental agencies. Individuals have incentives to open their wallets as well, if 
it is a prerequisite for receiving tax refunds. However, ease of use should be a priority when designing the complete system in order to make the adoption of the system as convenient as possible for users, thereby reducing noncompliance problems.

Beyond the deliberations related to governance and managerial issues, technical issues invoked by a blockchain solution should also be considered. In general, blockchain is subject to security threats whenever a single entity holds $51 \%$ of the computing power. A $51 \%$ attack is considered to be the worst-case scenario, since attackers would be able to claim all transactions for themselves (Yli-Huumo et al. 2016). Several approaches exist to reduce this threat including share chains (i.e., decentralized peer-to-peer miner networks) and a non-outsourceable proof of work (Tschorsch and Scheuermann 2015). However, currently the threat presented by large computing power prevails. Furthermore, privacy issues could arise through potential pseudonymity breaks created by profiling in the course of tracking transactions (Kaminsky 2011). Currently, these issues are addressed by third-party transaction pooling services that disguise the flow of transactions (Juels et al. 2016). However, sophisticated network analytical approaches can still lead to the identification of individual users (Tschorsch and Scheuermann 2015). Other privacy concerns could arise from the potentially unlimited storage of all blockchain transactions that could violate new EU privacy regulations. However, in blockchain databases block pruning renders nodes preceding a certain point unreadable and reduces the required amount of data storage (Buntinx 2015). Thus, we consider the latter privacy issue to be of minor relevance.

Common blockchain limitations also concern the throughput, latency, size, and bandwidth of transactions (Yli-Huumo et al. 2016). Some researchers discard these concerns as transient inefficiencies which will soon be overcome (Glaser 2017), or argue that thin clients might already mitigate these scalability issues (Tschorsch and Scheuermann 2015). In the present context, we consider scalability concerns to be of minor relevance since dividend payments only occur periodically, are not frequently exchanged but rather transferred to a final recipient, and are not subject to time critical transactions, for example, as bitcoin exchanges. Other practical concerns might address the cost of the system. While the price of the blockchaintoken Ether, for example, is subject to changes, the exchange rate of Ether to the dividend can be stored in the metainformation of the token. Thus, the system could link the amount of end-user tax refund entitlement to the actual value at the point of dividend payment.

Lastly, difficulties in correcting errors in data entries must also be considered. While the immutable log of transactions enabling fraud traceability is generally considered to be the key benefit of a blockchain database in this context, it also poses challenges when trying to correct a careless mistake. Comparable to any other type of erroneous money transfer, an incorrect token transfer must be corrected manually after the fact. However, we would argue that having an immutable transfer log should actually facilitate reclaiming the unintentional transaction. In the case of major errors in the code, a large percentage of miners would have to agree on a fork that dates back to a point in time preceding the triggering event and implement a corrected algorithm (Tschorsch and Scheuermann 2015). Thus, overall it can be seen that while blockchain offers certain benefits for overcoming the double spending issue compared to traditional database solutions, it also introduces some uncertainties which must be overcome by future developments.

\section{Conclusion}

The goal of this paper was to critically assess the potential of blockchain as a solution for managing dividend flows in order to overcome the current double spending problem in the public taxation sector. Double spending refers to the public authorities' current problem of refunding illegitimate tax claims in multinational dividend payment situations. At the moment there is no system implemented to provide tax agents with the information necessary to properly assess an applicant's eligibility for a tax refund. In light of the current absence of a database and considering the guidelines provided by the blockchain application framework (Glaser 2017), we determined that this issue presents a relevant use case for a blockchain database. Beyond the technical feasibility, blockchain also seems to be legally applicable to this case in light of the recent advances of blockchain-based transactions in the public service sector (e.g., approval and distribution of public welfare). Compared to traditional database systems, blockchain provides a comprehensive solution (i.e., on infrastructure, application and presentation levels) that can be adapted with relatively less effort by other stakeholders (e.g., other tax authorities, financial institutions, individual users). Conclusively, the blockchain's immutable log of historical transactions prevents banks from submitting erroneous reports and enables swift retraction of transactions in order to detect fraudulent applications.

In more detail, the proposed blockchain-based system requires VP Securities - as a trusted party - to issue a number of tokens into a blockchain database that corresponds to the amount of the originally distributed dividend. These tokens are transferred in the blockchain parallel to the cash flow. Thus, the ultimate dividend recipient also receives the respective amount of tokens, which can be 
redeemed for a tax refund with the tax authorities. By limiting the tokens to the amount of distributed dividend, we prevent the rampant tax refund claims of unentitled institutions and individuals. Moreover, the transaction logging from the initial token-issuing organization up to the entity ultimately receiving the dividend enables tax authorities to overcome the lack in transaction transparency in the dividend payment system.

Thereby, our design science approach contributes to the growing field of blockchain research. As reported by Ølnes (2016), scientific blockchain research has predominantly been limited to cryptocurrencies, especially Bitcoin. Our design science approach extends previous research by linking digital dividend cash flows with physical transactions. In doing so, we provide complementary insights into the role of blockchain as an intermediary technology, thus facilitating the possibility of transactions beyond the management of digital assets, marketplaces, and notary services (Korpela et al. 2017; Wörner et al. 2016), supply chain information (Korpela et al. 2017), or providing decentralized services in the energy (Aitzhan and Svetinovic 2016) or healthcare sectors (Mettler 2016). Moreover, this research is among the first design science approaches providing scientifically validated information on how to successfully implement blockchain-based applications (Beck et al. 2016). Future design science approaches can build upon our evaluated model when pursuing this promising field of research.

By developing this use case, our work also offers practical contributions demonstrating the business value of the potentially disruptive blockchain technology. First and foremost our prototype was developed and evaluated in the public service sector. Thus, it is of immediate relevance to all taxation authorities struggling to overcome the double spending issue (e.g., in the European Union). Considering that this problem has caused 1.8 billion USD damage to the Danish tax payers alone, our system can be considered to be of substantial practical relevance in this context (Skatteministeriet 2016). However, the logic of our design is not limited to the Danish tax system alone. Such a system could replace any of the existing intermediary banking systems (i.e., bank transaction and account managing systems) employed by companies for various services. Thus, this blockchain-based approach holds tremendous cost-saving potential for all larger companies that pay for these intermediary services. Moreover, due to the immutable transaction logging, erroneous payments can be easily corrected and compliance with auditing guidelines can be monitored and controlled. Furthermore, we provide the first practical evidence for the applicability of blockchain technology in the public sector - which could be easily expanded to various other fields, such as European VAT system or disintermediated NGO donations (Government Office for Science 2016). Thus, this prototype represents a first viable approach towards practical issues of public tax administrations.

\subsection{Limitations and Future Work}

The contribution of this study must be considered in light of its limitations, which also build the basis for future research. First, the generalizability of our work is limited regarding the applied context. While we argue that this prototype could be applied to other public services (e.g., European VAT or NGO donations) and the internal corporate finance environment - replacing and improving established banking systems because of the functional comparability of the systems - we only developed and evaluated it with employees of the Danish tax authority. Without further testing, our prototype is only applicable to the Danish tax authority as a valid approach for overcoming the targeted national double spending issue. As a next step, we would implement the second phase of the development of a tax refund application system in collaboration with SKAT. External tax authorities would be added as users to allow them to confirm applicant residence, and to conveniently perform lookups in a user role similar to SKAT's, which would incentivize collaboration. In developing the system further, usability should be a high priority to avoid discouraging users from adopting the system. As soon as a first system is in operation and tested, future research will be able to introduce these systems within corporations. Finally, the present blockchain-based solution is subject to certain practical limitations (e.g., scalability, privacy, cost efficiency) as elaborated earlier. While this study did not focus on eliminating these blockchain-inherent issues, we join others (Tschorsch and Scheuermann 2015; Yli-Huumo et al. 2016) in calling for research to prospectively overcome these problems.

\section{References}

Aitzhan NZ, Davor S (2016) Security and privacy in decentralized energy trading through multi-signatures, blockchain and anonymous Mmssaging streams. IEEE Trans Dependable Secur Comput

Avital M, Roman B, John LK, Matti R, Robin T (2016) Jumping on the blockchain bandwagon: lessons of the past and outlook to the future. In: Proceedings of the 37th international conference on information systems, Dublin

Bank of International Settlements (2017) Distributed ledger technology in payment, clearing and settlement: an analytical framework. Bank of International Settlements

Beck R, Müller-Bloch C (2017) Blockchain as radical innovation: a framework for engaging with distributed ledgers. In: Proceedings of the 50th Hawaii international conference on system sciences, Waikoloa Village

Beck R, Czepluch JS, Lollike N, Malone S (2016) Blockchain - the gateway to trust-free cryptographic transactions. In: Proceedings 
of the 24th European conference on information systems, Istanbul

Bennett K (1995) Legacy systems: coping with success. IEEE Softw 12(1):19-23

Buntinx J-P (2015) Pro's and con's on bitcoin block pruning. https:// news.bitcoin.com/pros-and-cons-on-bitcoin-block-pruning/. Accessed 28 Apr 2017

Carlström V (2016) Central Bank of Denmark is considering an e-krone based on blockchain - but tech is the least of the problems involved. http://nordic.businessinsider.com/the-danish-central-bankis-considering-an-ekrona-based-on-blockchain-but-privacy-couldbe-a-problem-2016-12/. Accessed 28 Apr 2017

Department for Work and Pensions (2013) Fraud and error in the benefit system. https://www.gov.uk/government/collections/ fraud-and-error-in-the-benefit-system. Accessed 25 Dec 2016

Epstein J (2015) Can blockchain technology reduce third-world poverty? A better way to keep track of who owns what land. http://reason.com/blog/2016/04/30/bitfury-desoto-blockchainland-registry. Accessed 25 Dec 2016

Ethplorer (2017) Top 50 Ethereum tokens for 90 days. https:// ethplorer.io/top. Accessed 21 Jul 2017

Eureporter.co (2013) Fight against fraud: study confirms billions lost in 'VAT gap'. www.eureporter.co/economy/2013/09/20/fight-againstfraud-study-confirms-billions-lost-in-vat-gap/. Accessed 25 Dec 2016

Fabian B, Ermakova T, Sander U (2016) Anonymity in bitcoin? The users' perspective. In: Proceedings of the 24th European conference on information systems, Istanbul

Gervais A, Karame GO, Wüst K, Glykantzis V, Ritzdorf H, Capkun S (2016) On the security and performance of proof of work blockchains. In: Proceedings of the 2016 ACM SIGSAC conference on computer and communications security, ACM, pp 3-16

Glaser F (2017) Pervasive decentralisation of digital infrastructures: a framework for blockchain enabled system and use case analysis. In: Proceedings of the 50th Hawaii international conference on system sciences; Waikoloa Village

Government Office for Science (2016) Distributed ledger technology: beyond block chain. https://www.gov.uk/government/uploads/ system/uploads/attachment_data/file/492972/gs-16-1-distrib uted-ledger-technology.pdf. Accessed 25 Dec 2016

Hevner AR, March ST, Park J, Ram S (2004) Design science in information systems research. MIS Q 28(1):75-105

Juels A, Kosba A, Shi E (2016) The ring of Gyges: investigating the future of criminal smart contracts. In: Proceedings of the 2016 ACM SIGSAC conference on computer and communications security, ACM, pp 283-295

Kaminsky D (2011) Black ops of TCP/IP 2011. https://dankaminsky. com/2011/08/05/bo2k11/. Accessed 28 Apr 2017

Karame GO, Androulaki E, Capkun S (2012) Double-spending fast payments in bitcoin. In: Proceedings of the 2012 ACM conference on computer and communications security, ACM, pp 906-917

Korpela K, Hallikas J, Dahlberg T (2017) Digital supply chain transformation toward blockchain integration. In: Proceedings of the 50th Hawaii international conference on system sciences

Kosba A, Miller A, Shi E, Wen Z, Papamanthou C (2016) Hawk: the blockchain model of cryptography and privacy-preserving smart contracts. In: 2016 IEEE symposium on security and privacy, IEEE, pp 839-858

LeBeau Z (2017) What's the difference between an 'ICO' and a tToken launch'? https://medium.com/@SingularDTV/whats-thedifference-between-an-ico-and-a-token-launch-7105edbb2112. Accessed 18 Jul 2017

Matussek K (2016) Prosecutors open dividend-tax probe after Commerzbank report. https://www.bloomberg.com/news/articles/ 2016-05-09/prosecutors-open-dividend-tax-probe-after-commerz bank-reports. Accessed 28 Apr 2017

Mettler M (2016) Blockchain technology in healthcare: the revolution starts here. In: IEEE 18th international conference on e-health networking, applications and services, IEEE, pp 1-3

Moody DL (2003) The method evaluation model: a theoretical model for validating information systems design methods. In: Proceedings of the 11th European conference on information systems, Naples

Nakamoto S (2008) Bitcoin: a peer-to-peer electronic cash system. https://bitcoin.org/bitcoin.pdf. Accessed 16 Oct 2017

Natoli C, Gramoli V (2016) The blockchain anomaly. In: 15th International symposium on network computing and applications, IEEE, pp 310-317

Nofer M, Gomber P, Hinz O, Schiereck D (2017) Blockchain. Bus Inf Syst Eng 59(3):183-187

Offermann P, Blom S, Schönherr M, Bub U (2010) Artifact types in information systems design science - a literature review. In: International conference on design science research in information systems, Springer, New York, pp 77-92

Ølnes S (2016) Beyond bitcoin enabling smart government using blockchain technology. In: International conference on electronic government and the information systems perspective, Springer, New York, pp 253-264

Peffers K, Rothenberger M, Tuunanen T, Vaezi R (2012) Design science research evaluation. In: International conference on design science research in information systems, Springer, New York, pp 398-410

Peffers K, Tuunanen T, Rothenberger MA, Chatterjee S (2007) A design science research methodology for information systems research. J Manag Inf Syst 24(3):45-77

Pilkington M (2016) Blockchain technology: principles and applications. In: Olleros FX, Zhegu M (eds), Research handbook on digital transformations. Edward Elgar

PWC (2015) International tax news, edn 28, June 2015. http:// download.pwc.com/ie/pubs/2015-pwc-ireland-international-taxnews-june-2015.pdf. Accessed 25 Dec 2016

PWC (2016) Denmark-foreign tax relief and tax treaties. http:// taxsummaries.pwc.com/uk/taxsummaries/wwts.nsf/ID/Den mark-Individual-Foreign-tax-relief-and-tax-treaties. Accessed 25 Dec 2016

Rosenfeld M (2014) Analysis of hashrate-based double spending. arXiv preprint arXiv:1402.2009

Saldaña J (2009) The coding manual for qualitative researchers. Sage Skatteministeriet (2016) Endelig Opgørelse fra SKAT om Formodet Svindel med Udbytteskat. http://www.skm.dk/aktuelt/presse/ pressemeddelelser/2016/august/endelig-opgoerelse-fra-skat-omformodet-svindel-med-udbytteskat. Accessed 25 Dec 2016

Sompolinsky Y, Aviv Z (2015) Secure high-rate transaction processing in bitcoin. In: International conference on financial cryptography and data security, Springer, New York, pp 507-527

Tschorsch F, Scheuermann B (2015) Bitcoin and beyond: a technical survey on decentralized digital currencies. IEEE Commun Surv Tutor 18(3):2084-2123

Venable J, Pries-Heje J, Baskerville R (2016) FEDS: a framework for evaluation in design science research. Eur J Inf Syst 25(1):77-89

Williams-Grut O (2016) Estonia is using the technology behind bitcoin to secure 1 million health records. http://www.busines sinsider.de/guardtime-estonian-health-records-industrial-block chain-bitcoin-2016-3. Accessed 25 Dec 2016

Wörner D, Von Bomhard T, Schreier Y-P, Bilgeri D (2016) The bitcoin ecosystem: disruption beyond financial services? In: Proceedings of the 24th European conference on information systems, Istanbul

Yli-Huumo J, Ko D, Choi S, Park S, Smolander K (2016) Where is current research on blockchain technology? A systematic review. PLoS One 11(10):e0163477 